\title{
EL APRENDIZAJE DE LA BIOÉTICA BASADO EN PROBLEMAS (ABBP): UN NUEVO ENFOQUE PEDAGÓGICO
}

\author{
Fabio Alberto Garzón Díaz, Betty Zárate ${ }^{2}$
}

Resumen: El artículo es el resultado del proyecto de Investigación HUM-948, de la Línea de bioética, educación y cultura del Bioethics Group, Universidad Militar Nueva Granada, y presenta la propuesta: "Aprendizaje de la Bioética Basado en Problemas (ABBP)". El texto se compone de siete partes: (1) una introducción que plantea la educabilidad y enseñabilidad de la bioética; (2) un desarrollo de sus características principales; (3) los fundamentos psicopedagógicos del Aprendizaje Basado en Problemas (ABP); (4) sus criterios esenciales; (5) un análisis de sus fundamentos epistemológicos; (6) una propuesta sobre cómo se entendería el ABBP, y (7), a modo de propuesta, cinco aspectos que se debería tener en cuenta a la hora de plantear un currículo de bioética basado en problemas y competencias.

Palabras clave: aprendizaje, problemas, competencias, epistemología de segundo orden, bioética

Learning bioethics based on problems (LBBP): a new pedagogical focus

Abstract: This article results from research project HUM-948, about the field of bioethics, culture and education by Bioethics Group, Military University of New Granada, presenting the proposal: "Learning bioethics based on problems (LBBP)". The text is composed of seven parts: (1) an introduction posing the possibility of training and learning bioethics; (2) development of their main characteristics; (3) psycho pedagogic base of LBBP; (4) essential criteria; (5) analysis of epistemological base; (6) a proposal of how to understand LBBP; and (7) as a proposal, 5 issues which should be taken into account when planning a bioethics curricula based on problems and competencies.

Key words: learning, problems, competencies, second order epistemology, bioethics

\section{O Aprendizado da Bioética Baseado em Problemas (ABBP): um novo enfoque pedagógico}

Resumo: O artigo é resultado do projeto de Investigação HUM-948, da Linha de bioética, educação e cultura do Bioethics Group, Universidad Militar Nueva Granada, e apresenta a proposta: "Aprendizado da Bioética Baseado em Problemas (ABBP)". O texto se compóe de sete partes: (1) uma introdução na qual se propóe a educabilidade e ensinamento da bioética; (2) um desenvolvimento de suas características principais; (3) os fundamentos psicopedagógicos do ABP; (4) os seus critérios essenciais; (5) uma análise de seus fundamentos epistemológicos; (6) uma proposta sobre como entender o ABBP, e (7), a modo de proposta, cinco aspectos que se deveria ter em conta na hora de propor um currículo de bioética baseado em problemas e competências.

Palavras-chave: aprendizado, problemas, competências, epistemologia de segunda ordem, bioética

\footnotetext{
${ }^{1}$ Programa de Doctorado en Bioética de la Facultad de Educación y Humanidades de la Universidad Militar Nueva Granada, Bogotá, Colombia

Correspondencia: fabio.garzon@unimilitar.edu.co

${ }^{2}$ Facultad de Educación y Humanidades de la Universidad Militar Nueva Granada, Bogotá, Colombia
} 


\section{Introducción}

La bioética cumple sus primeros 42 años desde aquella intuición presentada por Potter en su famoso libro "Bioethics: bridge to the future" (1). En este tiempo se han realizado esfuerzos por determinar su estatuto epistemológico. Sin embargo, es necesario reconocer que ha encontrado su oikos en la crisis de las ciencias de la salud. Discusiones sobre problemas éticos del comienzo y final de la vida; la gestión del cuerpo y la autodeterminación por parte de los pacientes; la justicia y la distribución de los recursos en salud; la experimentación biomédica con seres humanos; los derechos humanos de primera y segunda generación, etc., han marcado, para muchos engañosamente, el rumbo de lo que hoy conocemos como bioética(2).

Aunque entendamos la bioética como una nueva disciplina o como sabiduría, no solo en su enfoque inter-multi-disciplinar, sino trans-disciplinar (escuela brasileña(3), o como una ética aplicada (al estilo Adela Cortina)(4), es claro que la bioética ha entrado con mucha fuerza en la Academia. En palabra de Couceiro: "la bioética es una nueva disciplina académica que ha sido, de entre todas las humanidades médicas, las que más se ha desarrollado, y probablemente la que exigen con gran urgencia los planes de estudios de medicina para dotar a sus alumnos de las competencias que les permitan manejar los conflictos de valores en una relación clínica auténticamente democrática”(5).

Esto nos lleva a las siguientes preguntas: ¿la bioética es enseñable? ¿Quién debe enseñar bioética? ¿Qué se debe enseñar en bioética? ¿Cómo se debe enseñar bioética? ¿Es solo para las ciencias de la salud? ¿Debe ser una asignatura obligatoria en los currículos de medicina?

En primer lugar, en todo proceso formativo intervienen por lo menos dos factores: la enseñanza y el aprendizaje. Educar consiste en enseñar algo a alguien, quien a su vez lo aprende(2). Lo importante es que estos dos procesos son totalmente activos y no como clásicamente se entendía el aprendizaje, esto es, como la recepción pasiva de información trasmitida por el docente. La educación no es solo un proceso de enseńanza sino también, y principalmente, un proceso de aprendizaje(2).
En todo ser humano hay tres procesos de aprendizaje distintos en tiempo, en ritmo y en la vía de adquisición: los conocimientos, las habilidades y las actitudes. No es clara aún la forma en que se aprenden esos procesos. Parece que se aprendieran primero las actitudes, después la habilidades y más tarde los conocimientos. Lo que se sabe hoy es que cualquier tipo de educación (ya sea en ciencias de la salud o en cualquiera profesión) debe apuntar a estos tres procesos de formación. Esto es lo que hoy conocemos como "educación por competencias". Epsein y Hundet entienden por "competencia" el conjunto de conocimientos (conocer y comprender), habilidades (saber cómo actuar) y actitudes humanas que permiten que un profesional desarrolle una excelente práctica laboral, adecuada al contexto social en el que se desarrolla(6). En conclusión, la competencia determina el grado de capacidad operativa del individuo en un entorno determinado(5). Lo importante, a la hora de definir cualquier competencia, es que cumpla tres condiciones: relevancia en el entorno profesional, transferibilidad al estudiante y posibilidad de ser evaluada objetivamente.

Ahora bien, la formación en bioética en los estudiantes de ciencias de la salud debería desarrollar estos tres elementos: conocimientos, habilidades y actitudes. Para Couceiro, "la enseńanza de la bioética a los estudiantes de medicina no puede ser ajena ni al paradigma educativo de las competencias, ni al desarrollo de sus elementos"(5). Y la misma Couceiro más adelante argumenta: "su enseñanza (la de la bioética) en las facultades de medicina debe insertarse en la misma lógica que la del resto de las materias, en el marco del currículo basado en competencias, y utilizando como metodología principal el aprendizaje basado en problemas" (5).

\section{El Aprendizaje Basado en Problemas (ABP)}

El aprendizaje basado en la resolución de problemas (ABP) se inició por primera vez en los años sesenta del siglo pasado en la Facultad de Medicina de la Universidad de McMaster (Ontario, Canadá) y poco después se fueron añadiendo otras facultades, como la de Newcastle (Australia), Southern (Illinois) y Maastrich (Holanda(7). En Estados Unidos, la Universidad de Nuevo México fue la primera en tener un programa académico 
con un currículo basado en este enfoque. En Latinoamérica, el enfoque lo aplican varias universidades, entre las que se cuentan la Universidad Estatal de Londrina y la Facultad de Medicina de Marília, en Brasil, la Universidad Autónoma de México y la Fundación Universitaria Sanitas, en Bogotá, entre otras. Para Dueñas, "en la actualidad otras universidades a través de sus escuelas han adoptado el enfoque pedagógico del ABP, bien sea total o parcial, en muy distintas áreas del conocimiento; diferentes organizaciones respaldan este enfoque y lo recomiendan como una herramienta útil en el proceso de enseñanza-aprendizaje" (8).

Pero, ¿qué entendemos por ABP? Barrows define el ABP como "un método de aprendizaje basado en el principio de usar problemas como punto de partida para la adquisición e integración de los nuevos conocimientos"(9). Las características fundamentales, según McMaster, son las siguientes:

El aprendizaje está centrado en el alumno: los estudiantes deben tomar la responsabilidad de su propio aprendizaje, identificando lo que necesitan investigar y conocer para tener un mejor entendimiento y manejo del problema que el tutor les ha planteado, determinando dónde conseguir la información necesaria (libros, revistas, bases de datos, etc.). De esta manera, se permite que cada estudiante personalice su proceso de aprendizaje, concentrándose en áreas limitadas del conocimiento.

El aprendizaje se produce en grupos pequeños de estudiantes: el ideal para implementar el ABP es trabajar con grupos conformados por seis a ocho estudiantes. El finalizar cada unidad pedagógica los estudiantes cambian aleatoriamente de grupo $\mathrm{y}$ trabajan con un nuevo tutor.

Los profesores son facilitadores (tutores) o guias: el tutor plantea preguntas a los estudiantes para que se cuestiones y encuentren por ellos mismos la mejor ruta de entendimiento y manejo del problema. Dentro del aula, eventualmente los estudiantes asumen este rol ellos mismos, exigiéndose así unos a otros. Los tutores no necesariamente deberán ser expertos, ya que podrían caer en la tentación de volver a la práctica tradicional de enseñanza, proporcionándole al alumno la infor- mación y respuesta directa a los problemas planteados.

Los problemas forman el foco de organización y estimulo para el aprendizaje: el problema representa el desafío que los estudiantes enfrentarán en la práctica real y proporciona la relevancia y la motivación para el aprendizaje. Los problemas en medicina normalmente usan diferentes formatos: paciente simulado, simulación por computador, historias clínicas, etc. Para entender el problema, los estudiantes deberán identificar sus necesidades, ya sea en ciencias básicas como en otras disciplinas. Así, el problema les da un enfoque para integrar información de muchas disciplinas. Todo esto facilita que posteriormente los estudiantes recuerden y apliquen en futuros pacientes lo aprendido.

Los problemas son el vehiculo para el desarrollo de habilidades de resolución de problemas clínicos: el problema planteado debe similar a como todo ocurre en el mundo real. Por ejemplo, en el caso de estudiantes de medicina, el problema debe presentar al paciente con la única información que este tiene: dolores y síntomas manifestados. Debe permitir que los estudiantes formulen preguntas al paciente (simulado), realicen exámenes físicos y ordenen análisis de laboratorio, y avancen además en el desarrollo de habilidades en la resolución de problemas, que seguramente tendrán cuando estén en su práctica clínica.

La nueva información se adquiere a través del aprendizaje autodirigido: si el currículo lo centramos en el estudiante y el profesor es el facilitador del aprendizaje, entonces esperamos que los estudiantes aprendan a partir del conocimiento del mundo real y de la acumulación de experiencia en virtud de su estudio e investigación. Durante este aprendizaje dirigido, los estudiantes trabajan juntos, discuten, comparan, revisan y debaten permanentemente lo que han aprendido(10).

\section{La fundamentación psicopedagógica del ABP}

La tesis fundamental de la psicología cognitiva es que el aprendizaje es un proceso de construcción de conocimiento, teniendo como base estructuras cognitivas y saberes ya establecidos(11). Se pueden establecer tres principios relacionados con 
el acto de aprender y los procesos implicados: el aprendizaje como proceso constructivo y no solamente receptivo; el proceso cognitivo llamado "metacognición" y su afectación por el uso, y los factores sociales y contextuales.

En un comienzo se pensó, desde la psicología conductista, que el estudiante almacenaba información en la memoria y luego la recuperaba, dependiendo de la calidad de la misma y de la necesidad de evocación voluntaria que se tuviese. La psicología cognitiva identificó posteriormente que una de las características de la memoria es su capacidad de asociación, de formar redes (la nueva información se integra a la existente, constituyendo redes semánticas).

El segundo aporte es que la metacognición afecta el aprendizaje. Este principio consiste en el monitoreo que hace el estudiante de sus propios procesos cognitivos, el establecimiento de metas, selección de estrategias y evaluación de logros. Las habilidades metacognitivas incorporan la capacidad del novato, es decir, del estudiante que recién ingresa al proceso de identificar sus propias conductas de autoaprendizaje, de modo que, cuando ya es un experto, solo afina y continúa evaluando y juzgando sus habilidades de pensamiento.

El tercer elemento que incide en el ABP corresponde a los factores sociales y contextuales que influyen en el aprovechamiento de los conocimientos. Para alcanzar este propósito, Gijselaers ha propuesto algunas estrategias, iniciando con las indicaciones que deben darse en condiciones problemáticas complejas, que sean significativas para los estudiantes y permitan obtener la solución desde diferentes perspectivas(12); por otra parte, Glaser señala que los factores sociales tienen influencia en el aprendizaje del estudiante. El trabajo en grupos y la exposición muestran diversos puntos de vista alternativos que generan desafíos de comprensión en los estudiantes(11), lo que resulta indispensable para lograr aprendizajes significativos, establecer relaciones fuertes y sustantivas entre lo que ya se sabe y los nuevos saberes, la comprensión entre la información recibida y el significado de la misma. Para el logro de esta comprensión, los sujetos deben haber revisado, modificado y enriquecido sus estructuras de pensamiento, estableciendo nuevas conexiones(13).
El ABP promueve la disposición afectiva y la motivación de los alumnos, condiciones indispensables para el logro de aprendizajes significativos. De esta manera, la tarea del docente es asegurar la suficiente movilización afectiva y volitiva del estudiante para que esté dispuesto al aprendizaje.

El ABP provoca conflictos cognitivos, haciendo que estos sean duraderos, ya que el organismo está buscando siempre un estado de equilibrio (homeostasis). Por eso, este conflicto se convierte en el motor afectivo indispensable para alcanzar conocimientos duraderos y significativos. Lo anterior muestra que la estrategia no es resultado de un trabajo solitario del estudiante. Para Vigotsky(14), el aprendizaje es una actividad social, a partir del intercambio de ideas con otros, que permite aportar ángulos diferentes frente a un problema. El tutor es fundamental en este proceso, ya que debe ser el mediador y potenciador del aprendizaje.

\section{Criterios esenciales del ABP}

El modelo de aprendizaje es funcional, se basa en solución de problemas, para lo cual se requiere el trabajo con otros y para otros.

El estudiante recibe guía y apoyo de sus pares. El aprendizaje es multidireccional entre los sujetos y las circunstancias objeto de aprendizaje; además, el concepto de espacio de aprendizaje cerrado (aula de clase) desaparece y se determinan ambientes de aprendizaje práctico, con escenarios disponibles para la consulta y resolución de inquietudes.

El aprendizaje está centrado en el estudiante. El $\mathrm{ABP}$ requiere una disposición para trabajar en grupo; tolerancia para enfrentarse a situaciones ambiguas; destrezas para la interacción personal, tanto intelectual como personal, y aún más en la esfera de lo emotivo; desarrollo de la imaginación y la creatividad; habilidad para comprender problemas y establecer adecuados procesos de comunicación; actuación desde un pensamiento crítico, reflexivo, imaginativo y sensitivo, entre otros. Es así que se cambian algunos paradigmas como:

1. De la instrucción a la construcción: conocer es un proceso de constante cambio, de estudiar 
la realidad cambiante desde diferentes perspectivas, lo que conduce a la transformación constante de aprehensión del conocimiento, además de la transformación del conocimiento mismo. Esa reestructuración cognitiva se da mediante el uso de un pensamiento activo y propio de las estructuras mentales del sujeto que puede y quiere conocer. Por ello, desde esta perspectiva, aprender lleva a la experimentación, a la resolución de problemas, a entender que no se está ajeno a los errores, sino que estos son el punto de partida de nuevos saberes que se integran a los preexistentes(15).

2. Del esfuerzo al interés: aprender haciendo y realizando actividades que interesan y llevan al descubrimiento tiene como resultado que el estudiante comprenda mejor y aprenda mejor. Esto conduce a que los docentes o tutores se enteren de lo que interesa a sus estudiantes, construyendo actividades para apoyar e incrementar esos intereses que, con el tiempo, se convierten en necesidades y se proyectan como proyectos de autoaprendizaje. Por ello, las propuestas de aprendizaje mediado, usando por ejemplo modalidades como B-learning - en todos y cada uno de sus componentes, estableciendo espacios de discusión frente a un tema- permiten la pluralidad de puntos de vista, enriqueciendo así la construcción y el interés por aprender.

3. De la obediencia a la autonomía: al comienzo del artículo presentábamos los modelos o enfoques pedagógicos, recordando que los primeros buscan un estudiante obediente, que de cuenta de lo aprendido en un proceso de memorización, en los que la sumisión es total al que tiene el saber, es decir, al profesor. $\mathrm{El} A B P$, en cambio, desarrolla mentes libres, reflexivas, tolerantes y críticas de sí mismas y de su entorno.

\section{Fundamentación epistemológica del ABP}

El aprendizaje tradicional se basa en procesos cognitivos de tipo lineal, esto es, aprendizaje condensado en tres elementos: exposición de contenidos, aprendizaje de la información y aplicación de lo aprendido a situaciones reales o ficticias. En cambio, el ABP desarrolla un aprendizaje cíclico, en el cual el centro es la generación de nuevo conocimiento a partir de situaciones problemáticas reales.

La pregunta de fondo es: ¿qué epistemología está detrás del ABP? Nos preguntamos, finalmente, por el conocimiento: ¿cómo los seres humanos conocen? Nos preguntamos por la relación clásica sujeto (que conoce) y objeto (por conocer). Para Sotolongo y Delgado, "los seres humanos nos hemos percibido como 'sujetos' de saber (más o menos en contraposición a los 'objetos' por conocer) solo a partir de los albores de esa Modernidad"(16):

i. La figura de René Descartes representa el modelo de la modernidad. Descartes separó claramente el sujeto de cognición (res cogitans) del objeto por conocer (res extensa). Como nos comenta Sotolongo y Delgado: "La "bipolaridad" inherente a esta figura epistemológica de la relación objeto-sujeto convertida en 'clásica' para la modernidad es la que ha condicionado una suerte de 'oscilación' pendular muy característica del pensamiento moderno que, o bien pone en juego dicha figura desde posiciones epistemológicas objetivantes (gnoseologizantes), que privilegian desmedidamente al objeto en su relación con el sujeto; o bien lo hacen desde posiciones epistemológicas subjetivantes (fenomenologizantes), que privilegian desmedidamente al sujeto en su relación con el objeto"(16):

Epistemologias que privilegian el objeto: el objeto pretende quedar "reflejado" tal cual es, sin la intervención del sujeto (sujeto que "no añade nada nuevo" a la realidad que se indaga, pues sus sensaciones y percepciones se limitan a "reflejar" las propiedades de los objetos indagados).

Epistemologías que privilegian el sujeto: el objeto queda reducido a un "fenómeno" susceptible de "constituirse" como una unidad de sentido en la conciencia del sujeto.

Epistemologias hermenéuticas: todo discurso humano "se nutre de una experiencia fundamental, que no es otra cosa que la mediación lingüística de la comprensión. La experiencia primigenia de la que se deriva toda experiencia está en el lengua- 
je, entendido no como un sistema estructural independiente de las condiciones de uso, sino, por el contrario, como las diversas lenguas históricas que cobran realidad y fisonomía en los distintos contextos enunciativos. Los métodos científicos son también modalidades del lenguaje que deben ser remitidos a las condiciones históricas de la enunciación. Por ello, el ideal de una racionalidad objetivante como criterio último de verdad, esto es, de una racionalidad que prescinde de la historicidad, debe ser relativizado, esto es, debe ser reconducido a las condiciones históricas del lenguaje. La experiencia fundamental de la lingüisticidad (Sprachlichkeit) pone en tela de juicio las pretensiones de verdad del ideal científico de racionalidad"(17).

ii. Las epistemologías de segundo orden plantean una nueva comprensión de la relación sujeto-objeto, mediante la mutación en el estatuto del sujeto, el redimensionamiento del objeto, la contextualización mutua, tanto del sujeto como del objeto, desde el contexto de la praxis cotidiana(16):

Estatuto del sujeto: los "sujetos del saber" no son, ni pueden ser, una suerte de "espejos" cognitivos que "reflejan" la realidad "tal cual ella es". Los sujetos no se pueden reducir únicamente a la "razón”, y tampoco esos sujetos son algo "ya listo y acabado" de una vez por todas, que tienen como misión aprehender "a posteriori" el mundo. Por el contrario, los sujetos son el resultado de un proceso de construcción (proceso nunca acabado) de subjetividades.

Redimensionamiento del objeto: objetos-del-saber no son, ni pueden ser, idénticos a las cosas mismas, son más bien el "resultado de los modelos o construcciones teóricas" que realizan los sujetos. Construcciones intersubjetivas mediadas por el lenguaje y el discurso. Además, los objetos (el mundo) tampoco está acabado ("esperando que los sujetos lo conozcan"), en cada instante el mundo se transforma (se complejiza más).

La mutua contextualización sujeto-objeto desde la praxis cotidiana: la relación clásica sujeto-objeto se ve mediada (a modo dialéctico) por la praxis cotidiana humana, que incorpora en sí misma los aspectos objetivos y subjetivos del quehacer cotidiano de hombres y mujeres sociales.
La "verdad" es la construcción de un consenso intersubjetivo acerca de una u otra interobjetividad investigada, a partir del contexto de praxis cognitiva en que están inmersos quienes la construyen(16). El ABP plantea un giro epistemológico: el paso de una epistemología de corte lineal (cartesiano) a una epistemología de segundo orden.

\section{Aprendizaje de la Bioética Basado en Proble- mas (ABBP)}

¿Cómo articular la enseñanza de la bioética, el currículo basado en competencias y el aprendizaje basado en problemas en un programa de medicina? Para Couceiro, "la enseñanza de la bioética en las facultades de medicina debe insertarse, como el resto de las asignaturas, en el marco de las competencias. Definir conocimientos y desarrollar habilidades y actitudes específicas es también el objetivo de esta disciplina”(5).

Es claro que la bioética debe proporcionar conocimientos ("estos conocimientos no son mera especulación sin relevancia práctica, sino que constituyen el andamiaje básico que hace posible la adquisición de habilidades")(5); desarrollar habilidades ("el alumno logrará así las competencias para actuar sobre los conflictos éticos que surgen en la vida diaria de las profesiones sanitarias, y también para analizarlos críticamente y tomar decisiones que sean éticamente consistentes") (5), y cambiar actitudes. Pero, ¿puede la enseñanza de la bioética cambiar actitudes? Como argumenta Couceiro, "la mayor parte de los autores ha contestado a esta cuestión negativamente, y no solo debido al peligro de intentar "adoctrinar" o manipular a las personas, sino también porque las actitudes fundamentales o el carácter moral de los estudiantes de medicina ya están formados cuando entran en la universidad"(5).

También es clásica la argumentación de Gregorio Marañón sobre el "médico bueno, o la bondad moral de la medicina”. Marañón profesó una ética de la virtud, entendida como el estudio de las actitudes, de acuerdo con el esquema virtudvicio(2). Para Marañón, es de suprema importancia la elección del futuro médico de acuerdo con sus actitudes; a esto es lo que llama "vocación". "La ética profesional brota, como una flor espontánea, de la vocación. Cuando el maestro 
descubre en el alumno la vocación verdadera y la conforta; y cuando en el terreno de la vocación demostrada siembra los conocimientos, está haciendo no solo un buen médico, sino un médico bueno, de profunda moral profesional. De aquí mi convicción, un tanto revolucionaria, de que no se precisan reglas de moral expresas ni cursos de deontología. En las facultades de medicina, la moral, como asignatura, no se enseña por lo común. Y eso, que escandaliza a algunos, tiene esta razón fundamental. El médico bien preparado en el sentido humano e integral que hemos expuesto, el médico de vocación y no el de pura técnica, ese no necesita de reglamentos para su rectitud. Al médico mal preparado, las reglas y consejos morales le serían perfectamente inútiles. Sobran aquí, como en todos los problemas de conducta moral, las leyes". (O.C, IX 348. Citado por Gracia D. 1998: 151).

Sin embargo, ya no estamos, como pensaba Marañón, en una ética de la virtud o de la vocación, estamos inmersos en una ética de los derechos: derechos humanos, derechos de los pacientes, derechos de los profesionales sanitarios, etc. Coincidimos con Couceiro al afirmar que un proceso formativo en bioética también transforma las actitudes y el carácter moral de las personas. Claro está, esa modificación es secundaria respecto del propio proceso de aprendizaje de conocimientos y habilidades, y no directamente inducida. Y concluye Couceiro diciendo: "el solo hecho de ser capaz de identificar los conflictos éticos de la práctica clínica, y de poder dar respuestas racionales y prudentes, conduce a que el alumno interiorice un procedimiento, una forma de actuación y una sensibilización ante los valores implicados en la relación clínica”(5).

Nos separamos del pensamiento de Couceiro en el cómo y el cuándo se debe enseñar bioética en un programa de medicina. Couceiro retoma la distinción clásica en la formación médica de dos ciclos de enseñanza: el básico y el clínico. Para nosotros es mejor llamarlo "ámbito universitario" y "ámbito clínico", ya que la distinción clásica entre básico y clínico nos ha llevado a que en los currículos de medicina se vea en los cuatro primeros semestres únicamente asignaturas básicas, sin ninguna relación con la clínica; y posteriormente, cuando el estudiante comienza su ciclo clínico, es incapaz de aplicar los conceptos aprendidos en la ciencias básicas a la resolución de problemas clínicos. Es tan marcado el problema que los profesionales que entran a hacer residencia en cualquier especialidad médica deben volver a tomar créditos y cursos en ciencias básicas.

Este problema lo combate el ABP desde el comienzo, implementando objetivos de aprendizaje centrados en casos, historias clínicas, pacientes simulados o cualquiera otra actividad que se asemeje a la situación real que se vive en la práctica clínica. Consideramos que la enseñanza de la bioética debe ser transversal a todo el currículo de los programas de medicina y no asignaturas sueltas o electivas en determinados semestres. Creemos que la mejor forma de enseñar bioética es comprender que el paciente es una unidad inseparable entre lo biológico, lo psicológico, lo social y lo ético. En este sentido, cuando el estudiante, por ejemplo, esté rotando por el Departamento de Pediatría y se le explique las patologías frecuentes del infante malformado, en ese momento debe entrar el tema de bioética y su ejercicio de identificar los conflictos éticos que se generan de esas patologías. Creemos que esta es la única forma para que el estudiante integre todos sus conocimientos y perciba al paciente como una unidad bio-sico-social y ética. De otra manera, siempre lo percibirá escindido: por un lado su componente biológico y por otro, y con mucha menor importancia, sus componentes ético, social y cultural.

Para la elaboración de cada unidad pedagógica empleando el ABBP se debe tener en cuenta las siguientes etapas:

Objetivos de aprendizaje (resultados del aprendizaje): son una descripción explícita, en términos concretos, de lo que se espera que los estudiantes deban aprender o deban ser capaces de hacer como resultado del aprendizaje. Estos objetivos indican las circunstancias en las cuales se sabe que el estudiante ha adquirido las competencias establecidas por el programa (grado/asignatura/módulo o unidad de aprendizaje) (18). Los objetivos de aprendizaje deben ser relevantes a las competencias (pertinentes al perfil del titulado del programa), claros ( $\sin$ ambigüedades), factibles (realizables en el tiempo acordado) y evaluables (establecen las únicas bases de la evaluación del estudiante). 
Problemas/situaciones. ¿Qué es un "problema” en el ABP? Lo importante en el ABP son las características del "problema" que se utiliza. Se ha encontrado que un problema efectivo es aquel no estructurado, en el cual la información se presenta de forma progresiva y redactada de manera que propicia la discusión del grupo, e incluye algunas veces frases de controversia. También se ha observado que se estimula la motivación del estudiante cuando la situación o el problema contienen elementos con los que puede identificarse y, fundamentalmente, que reflejan la realidad del futuro mundo laboral(18).

Evaluación. ¿Cómo se evalúa en el ABP?: obviamente, esta es una de las áreas que presenta más dificultades si se pretende evaluar el conocimiento (adquisición, comprensión e utilización), las habilidades (tanto técnicas como de aprendizaje) y las actitudes (de gran relevancia hacia el comportamiento profesional y las relaciones interpersonales)(18).

El ABP pone énfasis en el aprendizaje autodirigido. Los estudiantes deben conocer claramente las pautas de evaluación. Estas deben ser exclusivamente las que se relacionan con los objetivos de aprendizaje del programa, e incluir ítems que permitan al estudiante contestar satisfactoriamente preguntas de evaluación del conocimiento como, asimismo, demostrar responsabilidad (avisar en caso de ausencia, respetar los horarios, llevar a cabo la búsqueda de información a la que se han comprometido); habilidades de aprendizaje (demostrar la capacidad de formular hipótesis explicativas de la situación, colaborar efectivamente para programar y llevar a cabo un plan de estudio, justificar la información recogida con la evidencia resultante de un análisis crítico y organizarse en la gestión del tiempo); habilidades de comunicación (presentar la información de forma ordenada, expresarse clara y concisamente); habilidades interpersonales (mostrar tolerancia con los colegas del grupo, esperar el momento apropiado para intervenir y saber escuchar) (18).

Cada Unidad Pedagógica debería desarrollarse de la siguiente forma:
Primera sesión:

Paso 1. Presentación del problema a la clase, la Unidad Pedagógica (10 minutos).

Paso 2. Trabajo en grupos pequeños con las instrucciones de discutir el problema e identificar objetivos de aprendizaje de interés para el grupo (20 minutos).

Paso 3. Informe de cada grupo al resto de la clase por un representante de cada grupo (20 minutos).

Paso 4. Selección de temas de estudio independiente o contribución del tutor (10 minutos). El tutor/facilitador recomienda aquellos temas que considera de estudio individual y aquellos en los que un profesor dará una clase, no expositiva pero conceptual.

Segunda sesión:

Sesión del tutor/facilitador que incluye tiempo para preguntas y aclaraciones.

Tercera sesión:

Paso 1. Trabajo en grupos (60 minutos). Desarrollo del portafolio. Los estudiantes discuten dentro del grupo lo aprendido e identifican áreas de dudas o nuevos temas que deben aprender relacionados con los objetivos de aprendizaje y relevantes al problema.

Paso 2. Informe de cada grupo al resto de la clase sobre los temas que necesitan aclaraciones y posibles nuevos temas que se pueden explorar (10 minutos).

\section{Cuarta sesión:}

Paso 1. Evaluación del aprendizaje de la Unidad Pedagógica.

Paso 2. Retroalimentación con el tutor/facilitador sobre el trabajo de la sesión anterior.

Quinta sesión:

Paso 1.Evaluación de la Unidad Pedagógica: evaluación formativa que contenga por lo menos: una autoevaluación de cada uno de los estudiantes, una coevaluación de sus compañeros, una eva- 
luación por el tutor/facilitador y una evaluación de las actividades propuesta en la Unidad Pedagógica: portafolio y evaluación.

Paso 2. Revisión de objetivos de aprendizaje cumplidos.

\section{A modo de propuesta}

La enseñanza-aprendizaje de la bioética basada en problemas en las facultades de medicina (y de ciencias de la salud) se fundamenta en cinco aspectos:

Debe ser transversal a todo el plan de estudios: no recomendamos dividirla en cursos específicos, ya sea en fase preclínica o clínica.

Debe estar integrada a las otras asignaturas del currículo: a modo de ejemplo, proponemos que, cuando los estudiantes estén rotando en neonatología, debe integrarse la toma de decisiones éticas en grandes prematuros, el papel de los padres del prematuro en la toma de decisiones, el encarnizamiento terapéutico en estos pacientes, etc.

Debe partir de problemas reales: el aula ha de convertirse en el "gran laboratorio", en el cual el estudiante experimente las mismas sensaciones, retos y decisiones que deberá vivir cuando esté en la consulta con su paciente.

Los problemas deben integrar todas las disciplinas: el máximo reto para esta propuesta está en plantear buenos problemas, en los que se articulen los diferentes saberes: clínicos, sociales, culturales, éticos, etc.

Finalmente, para que podamos formar "buenos médicos" y "médicos buenos", debemos contar con un equipo docente interdisciplinario, que sea creativo, trabaje en equipo y con sentido de la responsabilidad en la formación del médico que requiere la sociedad a la que va a servir.

\section{Agradecimientos}

Este trabajo se encuentra enmarcado bajo el proyecto de investigación vigente: "El Aprendizaje Basado en Problemas una propuesta pedagógica para la Educación Superior desde la Declaración de Bolonia y la Declaración mundial sobre Educación Superior en el siglo XXI. La bioética como programa piloto. Fase II", de la Línea de Investigación: bioética, educación y cultura de Bioethics Group. 


\section{Referencias}

1. Potter R. V. Bioethics: bridge to the future. Englewood Cliffs, NJ: Prentice-Hall; 1971.

2. Gracia D. Fundamentación y enseñanza de la Bioética. En: Ética y Vida. Estudios de Bioética. Santa Fe de Bogotá: Editorial El Búho; 1998.

3. Garrafa V. Epistemología de la Bioética: enfoque latino-americano. Revista Colombiana de Bioética. 2009; 4(1): 73-92.

4. Cortina A. Ética aplicada y democracia radical. Madrid: Tecnos; 1993.

5. Couceiro A. Enseñanza de la Bioética y planes de estudio basado en competencias. Educ Med. 2008; 11(2): 69-76.

6. Epstein R, Hundert E. Defining and assessing professional competence. JAMA 2002; 287: 226-35.

7. Pallie W, Carr DH. The McMaster medical education philosophy in theory, practice and historical perspective. Medical Teacher 1987; 9: 59-71.

8. Dueñas VH. El aprendizaje basado en problemas como enfoque pedagógico en la educación en salud. Colomb Med 2001; 32: 189-196.

9. Barrows HS. A Taxonomy of problem-based learning methods. Medical Education 1986; 20: 481-6.

10. Morales P, Landa V. Aprendizaje basado en problemas. Theoria 2004; 13: 145-155.

11. Glaser R. The Maturing of the relationship between the science of learning and cognition and educational practice. Learning and Instruction 1991; 1: 129-144.

12. Giselaers WH, (eds.) Brigning Problem Based to Higher Education. Theory and Practice. San Francisco: Jossey-Bass Publisher; 1996.

13. Coll C. Concepciones y tendencias actuales en psicología de la educación. In: Coll C, Palacios J, Marchesi A. (comp.) Desarrollo psicológico y educación. Vol 2. Psicología de la Educación Escolar. Madrid: Alianza Editorial; 2002.

14. Álvarez A. Educación y desarrollo: la teoría de Vigotsky y la zona de desarrollo próximo. In: Coll C, Palacios J, Marchesi A. (comp.) Desarrollo psicológico y educación. Vol 2. Psicología de la Educación Escolar. Madrid: Alianza Editorial; 2002.

15. Ausubel D. Psicología Educativa, un punto de vista cognoscitivo. México: Trillas; 1976.

16. Sotolongo P, Delgado C. La epistemología hermenéutica de segundo orden. En: La revolución contemporánea del saber y la complejidad social. Red CLACSO; 2006.

17. Bertorello A. El estatuto epistemológico de la racionalidad hermenéutica; 2000. Consultado en: http://es.scribd.com/ doc/148317490/Racionalidad-hermeneutica-doc\#scribd

18. Branda L. El aprendizaje basado en problemas. De herejía artificial a res popularis. Educ Med 2009; 12(1): 11-23.

Recibido: 20 de agosto de 2013

Aceptado: 10 de septiembre de 2013 